\title{
Stretchable Conductive Fibers of Ultra-high Tensile Strain and Stable Conductance Enabled by Worm-shape Graphene Microlayer
}

DOI:

10.1021/acs.nanolett.9b02862

\section{Document Version}

Accepted author manuscript

Link to publication record in Manchester Research Explorer

Citation for published version (APA):

Sun, F., Tian, M., Sun, X., Xu, T., Liu, X., Zhu, S., Zhang, X., \& Qu, L. (2019). Stretchable Conductive Fibers of Ultra-high Tensile Strain and Stable Conductance Enabled by Worm-shape Graphene Microlayer. Nano Letters, 19(9), 6592-6599. https://doi.org/10.1021/acs.nanolett.9b02862

\section{Published in:}

Nano Letters

\section{Citing this paper}

Please note that where the full-text provided on Manchester Research Explorer is the Author Accepted Manuscript or Proof version this may differ from the final Published version. If citing, it is advised that you check and use the publisher's definitive version.

\section{General rights}

Copyright and moral rights for the publications made accessible in the Research Explorer are retained by the authors and/or other copyright owners and it is a condition of accessing publications that users recognise and abide by the legal requirements associated with these rights.

\section{Takedown policy}

If you believe that this document breaches copyright please refer to the University of Manchester's Takedown Procedures [http://man.ac.uk/04Y6Bo] or contact uml.scholarlycommunications@manchester.ac.uk providing relevant details, so we can investigate your claim.

\section{OPEN ACCESS}




\title{
Stretchable Conductive Fibers of Ultra-high Tensile Strain and Stable Conductance Enabled by Worm-shape Graphene Microlayer
}

\author{
Fengqiang Sun ${ }^{\mathrm{a}, 1}$, Mingwei Tian ${ }^{\mathrm{a}, 1,{ }^{*}}$, Xuantong Sun ${ }^{\mathrm{d}, 1}$, Tailin $\mathrm{Xu}^{\mathrm{b},{ }^{*}}$, Xuqing Liu ${ }^{\mathrm{d}}$, \\ Shifeng Zhu ${ }^{\mathrm{a}}$, Xueji Zhang ${ }^{\mathrm{c},}$, , Lijun Qu ${ }^{\mathrm{a},{ }^{*}}$
}

a Research Center for Intelligent and Wearable Technology, College of Textiles and Clothing, State Key Laboratory of Bio-Fibers and Eco-Textiles, Collaborative Innovation Center for Eco-Textiles of Shandong Province, Qingdao University, Qingdao, Shandong, 266071, P.R. China

${ }^{\mathrm{b}}$ Research Center for Bioengineering and Sensing Technology, University of Science and Technology Beijing, 30 Xueyuan Road, Beijing 100083, P. R. China c School of Biomedical Engineering, Shenzhen University Health Science Center, Shenzhen, Guangdong 518060, P.R.China

d School of Materials, The University of Manchester, Oxford Road, Manchester, M13 9PL, U.K.

\footnotetext{
${ }^{1}$ These authors equally contributed to this work Corresponding author: mwtian@qdu.edu.cn (Prof. Tian), xutailin@ustb.edu.cn (Prof. Xu), zhangxueji@ustb.edu.cn (Prof. Zhang), lijunqu@qdu.edu.cn (Prof. Qu)
} 
1 conductance is essential for wearable and stretchable device, to ensure the

2 performance of wearable devices is stable. Inspired by the peristaltic behavior of

3 arthropods, we designed a graphene coating similar to the caterpillar structure on the

4 PU fiber surface, enabled by coating the worm-shape graphene microlayer onto

5 polyurethane filaments. Such worm-shape filaments can be stretched up to $1010 \%$

6 with wide reversible electro-response range $\left(0<{ }_{\varepsilon}<815 \%\right)$, long-term durability ( $>4000$

7 stretching/releasing cycles), good initial conductivity $\left(\sigma_{0}=124 \mathrm{~S} \mathrm{~m}^{-1}\right)$ and high-quality

8 factor $(Q=11.26)$. Remarkably, the worm-shape filaments show distinctive

9 strain-insensitive behavior $\left(\Delta R / R_{0}<0.1\right)$ up to $220 \%$ strain. Furthermore, the filaments

10 as electrical circuits of LED to track signals from robust human joint movements are

11 also demonstrated for practical application. Such worm-shape filaments with

12 distinctive strain-insensitive behavior provide a direct pathway for stretchy

13 electronics.

14 Keywords: graphene, bionic, strain-insensitive, polyurethane filaments, stretchy 15 electronic. 
Smart wearable device, as an important case of artificial intelligence, is a

2 complex micro-system composed of electronic skin, ${ }^{1-3}$ actuators, ${ }^{4-6}$ stretchable

3 electronic circuits, ${ }^{7-9}$ flexible supercapacitors ${ }^{10-12}$ and strain sensors ${ }^{13-15}$ etc. The

4 conductivity of most of reported stretchable conductive fibers will changed if be

5 stretched or strained, and these conductive fibers could be suitable as sensors. But

6 stable conductance is essential for wearable and stretchable device, to ensure the

7 performance of wearable devices is stable. Electronic circuit is one kind of essential

8 electronic element to transfer electrical signal, which usually receives less awareness

9 and attentions for wearable devices. However, under various robust body movements,

10 commercial metal electronic circuits might affect or even change the electrical signal

11 of smart clothes and wearable devices. ${ }^{16-18}$ Besides, such rigid circuits could also

12 restrict limb movements and unsatisfied wearing experience. Therefore, stretchable

13 electronic circuits with high stretchability and strain-insensitivity are the desirable

14 electronic element for wearable devices and smart clothes.

A growing number of strain-insensitive stretchy electronics are reported for smart wearable devices. Currently, two-dimensional micro or nano films with invariant resistant changes are the mostly proposed strategies for stretchy electric circuits such as electrospun elastomeric mats decorated with silver nanoparticles, ${ }^{19}$ self-generated microfibril network on the SBS film, ${ }^{20}$ the fractal gold nanomesh-like structure, ${ }^{21}$ and stretchable wirings on Elastic substrate. ${ }^{22}$ However, these 2D stretchy electronics with various micro-patterns could only possess negligible resistant changes under low-level strains $(<100 \%)$, which is not suitable for tracking robust human joint 
1 movement (strains $>100 \%$ ). 1D fiber-shaped electronics might be the ideal structure

2 with high stretchability, Baughman et al. have reported 1D microlayer sheath-core

3 conducting fibers with microlayer buckling, ${ }^{23}$ enabling a resistance change of less

4 than $5 \%$ for a $1000 \%$ stretch of the rubber fibers, but such rubber fibers (ropes) with a

5 large diameter $1.7 \mathrm{~mm}$ might restrict the applications for smart clothes. Therefore, the

6 criterion for ideal fiber-shaped electronics generally includes small diameter,

7 strain-insensitivity under high-level strain and good durability.

8 Herein, inspired from the creeping of worms with an effective and logical

9 deformation, we fabricated the worm-shape filaments by coating worm-shape 10 graphene microlayer onto polyurethane filaments. Such bionic micro-structures 11 exhibit distinctive strain-insensitive behavior $\Delta R / R_{0}$ smaller than 0.1 up to $220 \%$ 12 stretch, along with long-term durability after 4000 stretching/releasing cycles, good 13 initial conductivity of $124 \mathrm{~S} \mathrm{~m}^{-1}$ and high-quality factor $(Q=11.26)$. Compared with 14 other stretchable electronics, inspired by the peristaltic behavior of arthropods, we 15 designed a graphene coating similar to the caterpillar structure on the PU filament surface. When stretched, our design is similar to the creep behavior, maintaining a stable electrical conductivity. The as-obtained worm-sharp graphene microlayer PU filaments exhibit ultra-high stretchable, excellent strain-insensitive and more suitable for wearable with better softness and comfort. Our worm-shape graphene microlayer polyurethane filaments open an avenue for the fabrication of seamless connection smart clothes and stretchable electronics.

\section{RESULTS AND DISCUSSION}


The processes for preparing of conductive polyurethane filaments with

2 worm-shape graphene microlayer are illustrated in Figure 1a. The graphene/PU (GP)

3 microlayer could be in-situ deposited on the surface of pristine PU filaments with the

4 effect of non-solvent-induced phase separation. ${ }^{24} \mathrm{GP}_{x} @$ filament denotes that GP

5 microlayer is deposited on a PU multifilament core under $x \%$ pre-stretching condition,

6 which is detailed in the experimental section. In brief, pristine PU filament was

7 stretched under the fabrication strain $x \%$, and then immersed into the graphene/PU

8 inks for capillary absorption, afterwards, the GP microlayer could be in-situ formed

9 on the soaked PU filaments in the coagulation water bath. After releasing the

10 pre-stretching forces loaded on the filament substrate, the worm-shape graphene

11 microlayer was achieved as the inset SEM of Figure 1a.

The diagram of the worm wriggle as in Figure $1 \mathrm{~b}$ indicates the prototypical model of the $\mathrm{GP}_{x} @$ filament. The as-prepared GP ${ }_{x} @$ filament exhibits remarkable elastic, which can be stretched over 800\% (GP $300 @$ filament). Furthermore, GP $300 @$ filament also exhibits strain-insensitivity as the stretchable electric circuits in Figure 1c, the luminance of LEDs can be scarcely changed with the effect of robust elongation of elastic circuits. The evolution trend of the GP microlayer on GP $300 @$ filament are shown in Figure 1d under strains from $0 \%$ to $250 \%$, the peak width tends to elongate while the peak height decrease owing to the flattening trend under the strain, and the peak unit would quickly recover to the original shape once releasing the strain. The structure of GP microlayer maintains well and the LED is always on even under $250 \%$ strain indicating the well strain-insensitive behavior under robust elongation. 
1

2

3

4

5

6

7

8

9

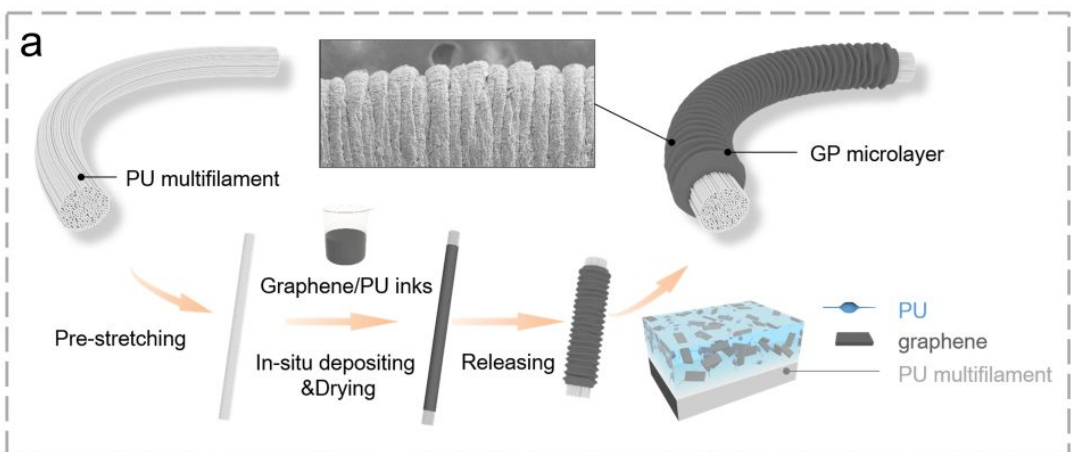

$\mathrm{b}$

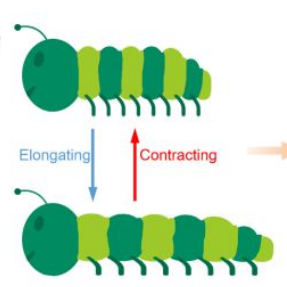

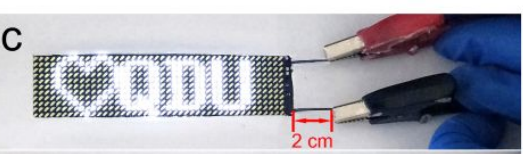

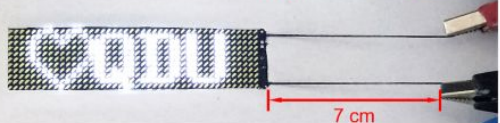

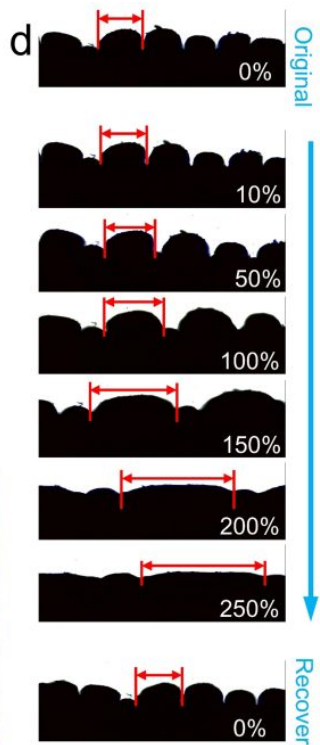

Figure 1. Bionic superelastic conductive polyurethane filaments with worm-shape graphene microlayer. (a) Schematic diagram of preparing the worm-shape filaments. (b) Prototypical model and photographs of a GP $300 @$ filament pull by 200 grams of weight. (c) LED patterned

lights response with different elongation of $\mathrm{GP}_{300} @$ filament circuits. (d) The optical images of GP microlayer of GP 300 @filament under stretching.

The morphology of GP $@ @$ filament and GP $300 @$ filament along with the pristine PU filament are illustrated in Figure 2a-f. From the longitudinal view of the filament, the bundle structure of $\mathrm{GP}_{0} @$ filaments (Figure 2c) is entirely covered by the GP microlayer, the increased diameter of $\mathrm{GP}_{0} @$ filament $(d=875 \pm 13 \mu \mathrm{m})$ compared with the pristine PU filament $(d=840 \pm 10 \mu \mathrm{m})$ implies the successfully deposited graphene microlayer. While the diameter of $\mathrm{GP}_{300} @$ filament $(d=775 \pm 22 \mu \mathrm{m})$ tends to be smaller due to the pre-stretching treatment and the contraction force from the GP layer. Under the higher magnification, the worm-shape graphene microlayer can be obviously observed on the surface of GP ${ }_{300} @$ filament while the $\mathrm{GP}_{0} @$ filament with 
1 smooth surface appearance. Furthermore, the graphene nanosheets are fixed stable on

2 the PU matrix from the inset of Figure 2c and Figure 2e. From the cross-section view

3 of the PU filament, the worm-shape graphene microlayer of GP $300 @$ filament exhibit a

4 thicker microlayer (thickness: $107 \pm 11 \mu \mathrm{m}$ ) than the smooth graphene microlayer of

$5 \quad$ GP $@$ filament (thickness: 74 \pm 7 $\mu \mathrm{m}$ ), and the GP microlayer is entirely deposited on

6 the surface of PU filament without obviously gap or pore indicating the well interface

7 interaction between the micro-layer and PU substrate.

8 In addition, the FTIR spectra of the as-obtained graphene, PU, and

$9 \mathrm{GP}_{300} @$ filaments are depicted in Figure 2g, only -OH group and the benzenoid $\mathrm{C}=\mathrm{C}$

10 stretching band appeared on graphene without any oxygen groups. In contrast, the

11 carboxyl absorption peaks and $\mathrm{C}-\mathrm{O}-\mathrm{C}$ and $\mathrm{C}-\mathrm{H}$ stretching bands of PU become

12 weaker indicating the sufficient blend of graphene and PU. ${ }^{25}$ The graphene was

13 obtained by lab-scale high-pressure homogenizing method and Figure $2 \mathrm{~h}$ shows the

14 orientation and exfoliation process preparing few-layer graphene in the flow path

15 during homogenizing process. The TEM images of the obtained graphene are

16 illustrated in Figure 2i. As shown in Figure 2i, the lateral size of the as-obtained

17 graphene is around $5 \sim 10 \mu \mathrm{m}$ and the graphene with few-layers can be clearly 18 observed. 

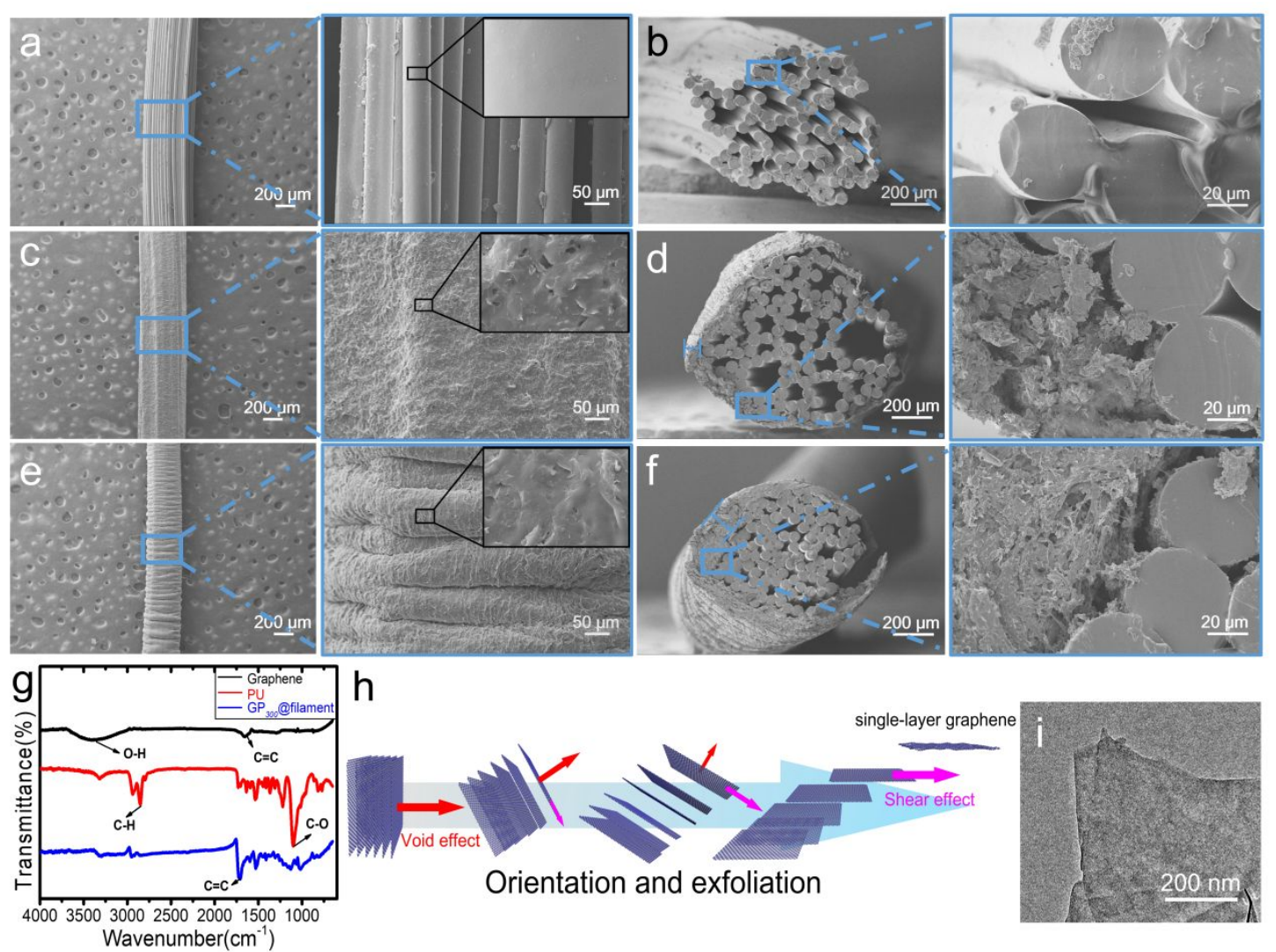

Figure 2. Morphology of the modified PU filaments, (a-f) SEM images of the pristine PU multifilament from longitudinal view (a) and cross section (b), GP 0 @filament (c) and (d), and GP $300 @$ filament (e) and (f). (g) FTIR spectra of graphene, PU, and GP $300 @$ filaments. (h)

Orientation and exfoliation process of lab-scale pressure homogenizing method preparing few-layer graphene in the flow path during homogenizing process. (i) TEM image of as-obtain few-layer graphene.

We further evaluate the mechanical and electrical properties of such filaments. As shown in Figure 3a, all the samples exhibit remarkable superelastic characteristic, the elongation at break of the $\mathrm{GP}_{x} @$ filament is gradually decreased with the increasing $x \%$ owing to the residual internal stress arising from the pre-stretching treatment. For example, the elongation of $\mathrm{GP}_{0} @$ filament is up to 1778\% and $\mathrm{GP}_{300} @$ filament is 
$1 \quad 1010 \%$. All the samples show almost the same breaking strength $(\sim 30 \mathrm{MPa})$ with low

2 modulus appearance. There are some random noise peaks on the stress-strain curve,

3 which resulted from the sudden breaking of the inside single PU filament.

4

5 illustrated in Figure 3b. All the samples exhibit distinctly different electrical responses

6 with increasing axial stretching process. For instance, GP $300 @$ filament exhibits the

7 widest reversible electroresponse range up to 815\% while GP 0 @filament could only

8 reach $50 \%$, therefore, the wide reversibility electroresponse range is the intrinsic merit

9 of the stretchy wearable electronics. Thereinto, the conductivity of $\mathrm{GP}_{300} @$ filament increases to $363 \mathrm{~S} \mathrm{~m}^{-1}$ at $\varepsilon=300 \%$ from $124 \mathrm{~S} \mathrm{~m}^{-1}$ at the free state $(\varepsilon=0 \%)$, and then quickly decreases to $28 \mathrm{~S} \mathrm{~m}^{-1}$ at $\varepsilon=800 \%$.

The variation of the initial electrical conductivity of the filament under $50 \%$ stretching cycling process is shown in Figure 3c, nearly all the GP ${ }_{x} @$ filaments exhibit steady appearance with the robust stretching cycles except GP 0 filaments. Furthermore, the strain dependence of relative resistance change $\Delta R / R_{0}$ is also evaluate (Figure 3d), for all the $\mathrm{GP}_{x} @$ filaments, relative resistance changes increase monotonically with increasing stretching process, and exhibit obviously lower rising rate with increasing pre-stretching $x \%$. Choosing $\Delta R / R_{0}=0.1$ as the baseline, GP $@ @$ filaments could quickly reach the baseline at 5\% while GP $300 @$ filaments at $220 \%$ indicating the outstanding strain-insensitive performance.

Herein, the quality factor $Q$ is employed to evaluate the electro-response of our filaments under various strain conditions, ${ }^{23}$ which is defined as the percent strain 
1 divided by the percent resistance change. Compared with previous reported

2 conducting coatings on elastomeric fibers or sheets, , 8, 26-31 All the GP ${ }_{x} @$ filaments

3 exhibit acceptable $Q$ level as shown in Figure 3e. The value of $Q$ gradually increases

4 from 4.62 of $\mathrm{GP}_{50} @$ filaments to 11.26 of $\mathrm{GP}_{300} @$ filaments, which are all higher than

5 the value of $Q$ in the literature, indicating the outstanding strain-insensitive behaviors

6 of the worm-shaped electronic circuits. Furthermore, in order to show the distinct

7 function of the worm-shaped microlayer, a simple trial is carried out to monitor

8 relative resistance changes of $\mathrm{GP}_{300} @$ filament and $\mathrm{GP}_{0} @$ filament under periodic

9 loading and releasing process with various strain ratios (Figure 3f). It can be found

10 that the relative resistance change of $\mathrm{GP}_{300} @$ filament can be nearly negligible even at

$11300 \%$ strain (red line), in contrast, that of $\mathrm{GP}_{0} @$ filament is more sensitive and the

12 response range is within $50 \%$ strain (blue line). Such results reveal that pre-stretching

13 treatment is the critical treatment for the stretchy electronics with the

14 strain-insensitive properties.
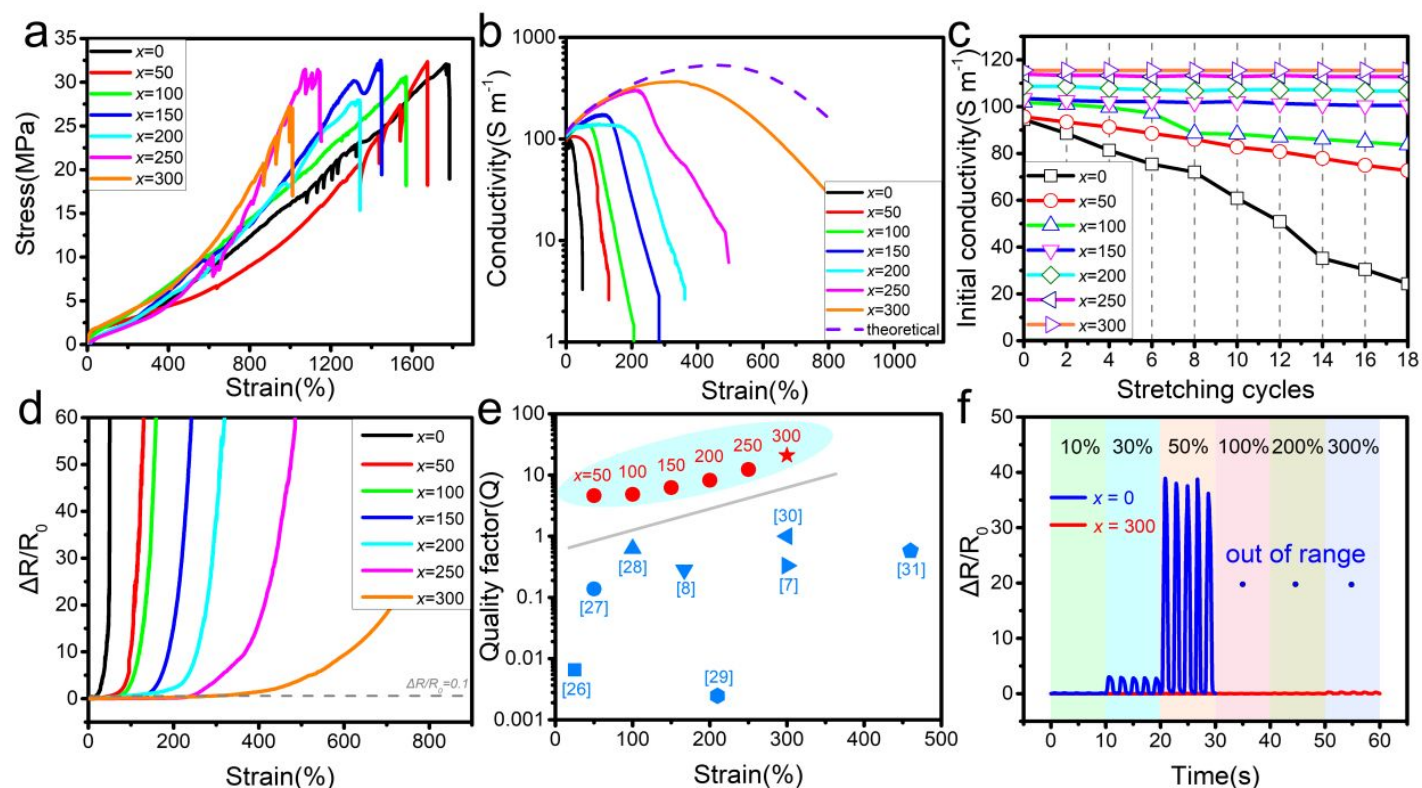

Figure 3. Mechanical and electrical properties of such filaments. (a) Conductivity-strain 
curves for GP $\mathrm{P}_{x} @$ filaments. (b) Conductivity-strain curves for GP $@$ @filaments and theoretical curves for $\mathrm{GP}_{300} @$ filament. (c) Variation of initial conductivity per centimeter during stretching-releasing cycles with 50\% strain. (d) Relative resistance curves of GP $\mathrm{P}_{x} @$ filaments. (e) Comparison of quality factor $Q$ for the GP ${ }_{x} @$ filaments with data in literature. (f) Dynamic relative resistance of GP $@ @$ filament and $\mathrm{GP}_{300} @$ ffilament under different strains with 2s cycle.

In order to further clarify the mechanism of electrical conductive of worm-shaped graphene microlayer, the micro geometrical model of GP microlayer unit on $\mathrm{GP}_{x} @$ filament during the stretching process is illustrated in Figure 4. The stretching process is classified into three simple stages, which can be divided by two boundary points. The contact critical point $\left(\varepsilon=\varepsilon_{\mathrm{c}}\right)$ is the special boundary that the two legs of the GP layer loop are point-contact, and the other boundary is the straighten pre-stretching state $(\varepsilon=x)$ that the strain degree matches with the pre-stretching state. Therefore, the tendency of the micro-structure of GP microlayer generally passes through three phases separated by these two boundary points: the original state to the contact critical point $\left(0<\varepsilon \leq \varepsilon_{\mathrm{c}}\right)$, the contact critical point to the straighten pre-stretching state $\left(\varepsilon_{\mathrm{c}}<\varepsilon \leq x\right)$, and after the pre-stretching state $(\varepsilon>x)$. The total resistance is expressed by an equation set which derived by Supplementary Method 1 and Supplementary Method 2. Based on this model, the initial value of $A, D_{0}$ and $l$ of $\mathrm{GP}_{x} @$ filament can be observed and calculated from Figure 2e, f. $R_{l}$ and $x$ are the variables which can be measured during the pre-stretching process. The free parameter $\left(\sigma_{\mathrm{d}}\right)$ is monotonously decreased to zero at $\varepsilon=\varepsilon_{\mathrm{c}}$. The electrical conductivity of $\mathrm{GP}_{300} @$ filament in Figure 3b 
1 could almost match such model, however, the total calculated conductivity is slightly

2 bigger than the measured conductivity, in the theoretical simulation, the hypothesis of

3 this model was PU multi-filaments as uniform cylinder and the graphene microlayer

4 as regular wrinkle layer. Actually, PU multi-filaments is consisted of single filaments

5 with unordered structure leading to complex deformation, so the mechanical

6 performance might be changed during stretching. In addition, the worm-shape

7 graphene microlayer is randomly generated by the inter stress of microlayer, so the

8 resultant wrinkle microlayer might be not so regular. Therefore, the discrepancy could

9 occurred between theoretical and experimental results, but such discrepancy is within

10 a reasonable margin of error.

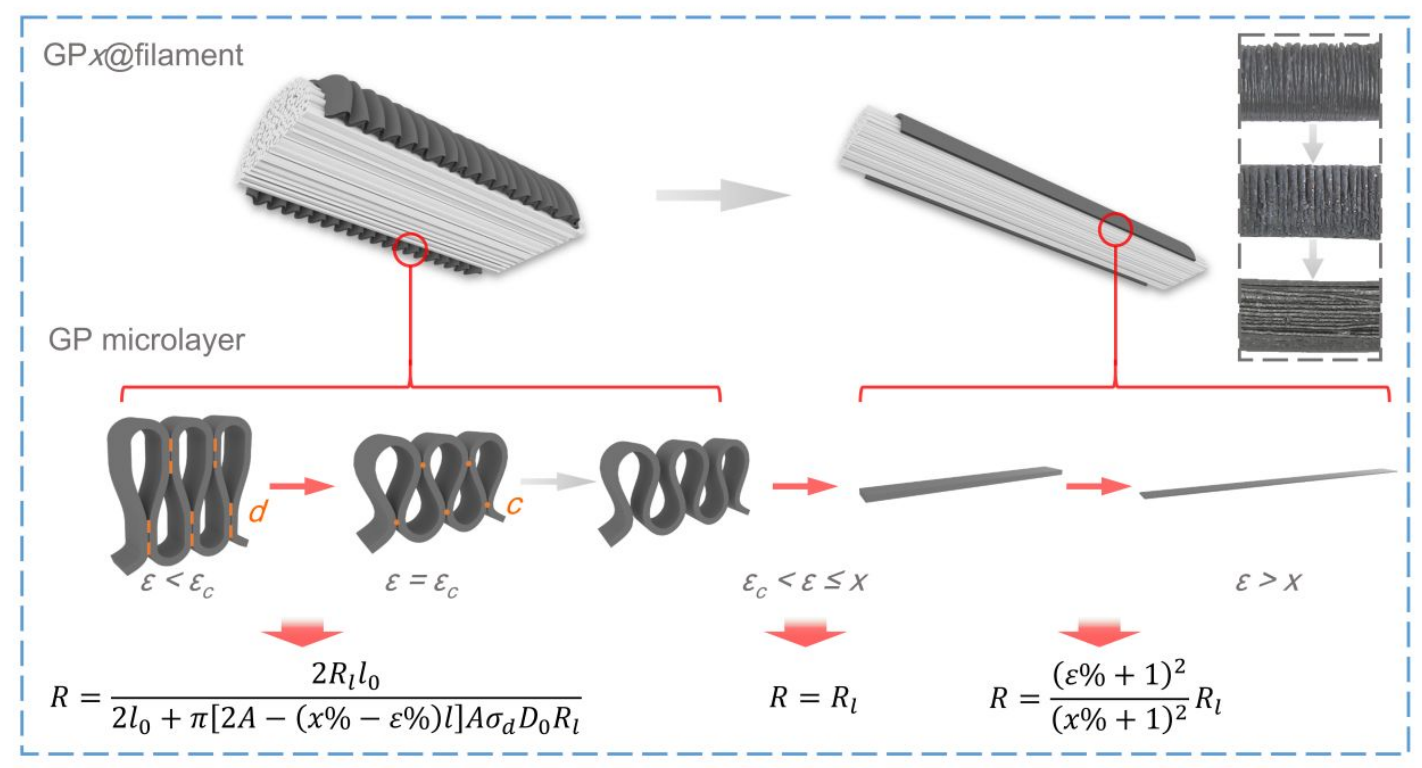

Figure 4. Geometrical model of GP microlayer unit on $\mathrm{GP}_{x} @$ filament and the corresponding theoretical formula at different stages during the stretching process.

Figure 5 explores the mechanical hysteresis and strain-insensitive properties of GP $300 @$ filament as example. GP $300 @$ filament exhibits slightly mechanical hysteresis 
1 and fine elastic recovery under different stretching cases from $20 \%$ to $140 \%$ in Figure

$25 \mathrm{a}$, indicating reliable resiliency. Figure $5 \mathrm{~b}$ shows the reversible relative resistance

3 change of $\mathrm{GP}_{300} @$ filament under the wide strain range $0 \sim 400 \%, \Delta R / R_{0}$ of the filament

4 is still under 0.8 even be elongated to $400 \%$ indicating the outstanding invariant

5 resistance changes under high level strain, and the hysteresis of the filament is as

6 small as 0.02 indicating the remarkable repeatability of $\mathrm{GP}_{300} @$ filament. The inset

7 exhibits tiny relative resistance change of 0.08 from $50 \%$ to $210 \%$ strain, which

8 reveals excellent stain-insensitive. Furthermore, the I-V curves is employed to

9 evaluate the electroresponse of the filaments under different stretching strain levels

10 (from 0 to $300 \%$ ). Under $5 \mathrm{~V}$ on-load voltage, the I-V curves (Figure $5 \mathrm{c}$ ) of the

11 filaments exhibit linear shape indicating the intrinsic properties of good conductor,

12 and the slope of these curves only decrease $21 \%$ for $300 \%$ strain compared with the

13 initial case, demonstrating excellent strain-insensitive and potential applications as

14 flexible electronic circuits. The durability and stability of $\mathrm{GP}_{300} @$ filament is

15 measured under the periodic loading/releasing cycles of $10 \%$ strain with 4000 cycles

16 in Figure 5d, the $\mathrm{GP}_{300} @$ filament could still possess good stability after 4000 cycles

17 with only $0.4 \%$ increasement of electrical resistance compared with the initial value.

18 The SEM images of PU filament before and after 4000 cycles are compared and

19 exhibit merely changes of the worm-shape graphene microlayer indicating excellent

20 durability of our PU filament. Furthermore, the abrasion property of GP $300 @$ filament

21 is also evaluated Figure S4. After 1000 times robust abrasion, the worm-shape

22 graphene micro-layer can still exist indicating good abrasion ability, but after 5000 
1 times robust abrasion, the microlayer was destroyed. According to the basic wearing

2 demand of textile, such abrasion performance is good enough for common wearing

3 textiles In addition, plucking is also a common action for human motion, so the

4 electroresponse of $\mathrm{GP}_{300} @$ filament under plucking deformation is shown in Figure 5e.

5 The filament was stretched under $141.4 \%$ strain with $45^{\circ}$ angle, and then was

6 suddenly released to the free state with attenuation vibration, the resultant relative

7 resistance changes is as low as 0.05 implying the low sensitivity to the external

8 stimulation. In a word, $\mathrm{GP}_{300} @$ filament reflect remarkable durable and stable

9 strain-insensitive behaviors, which can be applied for the potential wearable 10 electronic circuits.
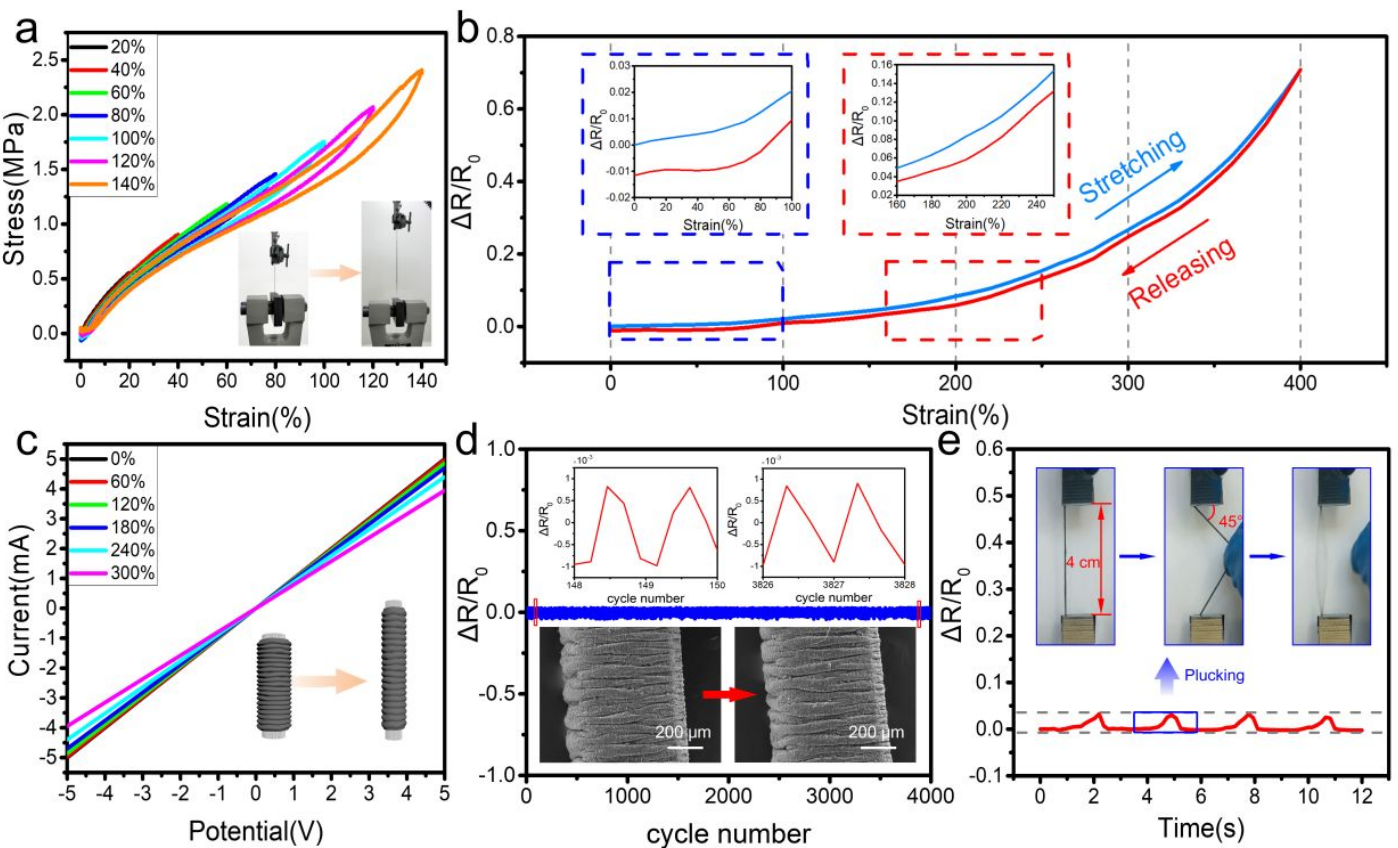

11

12

13

14

15

Figure 5. Strain-insensitive performance of such stretchy electronics. (a) Stress-strain curves of the GP ${ }_{300} @$ filament for different strains, showing hysteresis response during the stretching and releasing. (b) Relative resistance changes of $\mathrm{GP}_{300} @$ afilament as a function of stretching (blue line) and releasing (red line) within $400 \%$ strain. The insets show the relative resistance 
1 change at the range of $0 \%-100 \%$ and $160 \%-250 \%$ respectively. (c) $\mathrm{I}-\mathrm{V}$ curves of the

$2 \mathrm{GP}_{300} @$ filament at different stretching strain levels (from 0 to 300\%). (d) Durability and

3 stability test of $\mathrm{GP}_{300} @$ filament in the process of 10\% strain during 4000 loading/releasing

4 cycles, insets: the magnified diagram of the selected cycles, and SEM images of the

$5 \mathrm{GP}_{300} @$ filament before and after durability test. (e) Relative resistance changes of

$6 \mathrm{GP}_{300} @$ filament in the pluck of $141.4 \%$ strain.

7

To demonstrate the potential application of strain-insensitive GP $300 @$ filament as stretchy electronics. As the proof-of-concept, we design a series of preliminary trails. First, GP $0 @$ filament and GP $300 @$ filament are selected as the elastic electrical circuits for the weighing system at the same time in Figure 6a, $200 \mathrm{~g}$ weights is put on the pressure sensor, and two elastic circuits are separately stretched under $10 \%$ and $20 \%$ strain, and the $\Delta R / R_{0}$ curves of each circuits stretching are illustrated in Figure $6 \mathrm{~b}$ and the $\Delta R / R_{0}$ change of $\mathrm{GP}_{300} @$ filament is negligible while that of $\mathrm{GP}_{0} @$ filament reflects regularly changes under the periodic strain. The second trails are designed for an intuitive LED system to observe the luminance change of the LED when GP $@ @$ filament and $\mathrm{GP}_{300} @$ filament are stretched separately (Figure 6c, d and Supplementary Movie 1). The luminance of LED is barely changed with $300 \%$ strain of $\mathrm{GP}_{300} @$ filament, because $\Delta R / R_{0}$ is only 0.29 at $300 \%$ strain (Figure 6d). In contrast, when the GPo@filament is slightly stretched, the LED luminance immediately tend to darken at $16.7 \%$ strain and extinguished at $33 \%$ strain, due to the rapid increasing of $\Delta R / R_{0}$ (Figure 6d). 
1 Furthermore, the strain-insensitive GP $300 @$ filament is sewed into fabric substrate

2 to simulate various robust deformations such as stretch, knead, twist, and roll during

3 wearing or using (Figure 6e and Supplementary Movie 2). Besides, the fabrics

4 equipped with $\mathrm{GP}_{300} @$ filament could also be wore on human wrist, under the wrist

5 joint movement with various bending angles (Figure 6f, $g$ and Supplementary Movie

63 ), the resultant relative resistance changes is as low as 0.05 result in the luminance of

7 LED still remains brightness and is nearly no disturbed by the elongation of flexible

8 electronic circuits, with the aid of the remarkable the strain-insensitive of

$9 \mathrm{GP}_{300} @$ filament. Therefore, the superelastic conductive polyurethane filaments with

10 wore-shaped graphene microlayer can be acted as the recommendable candidate as

11 wearable electrical circuit for signal transmission. 

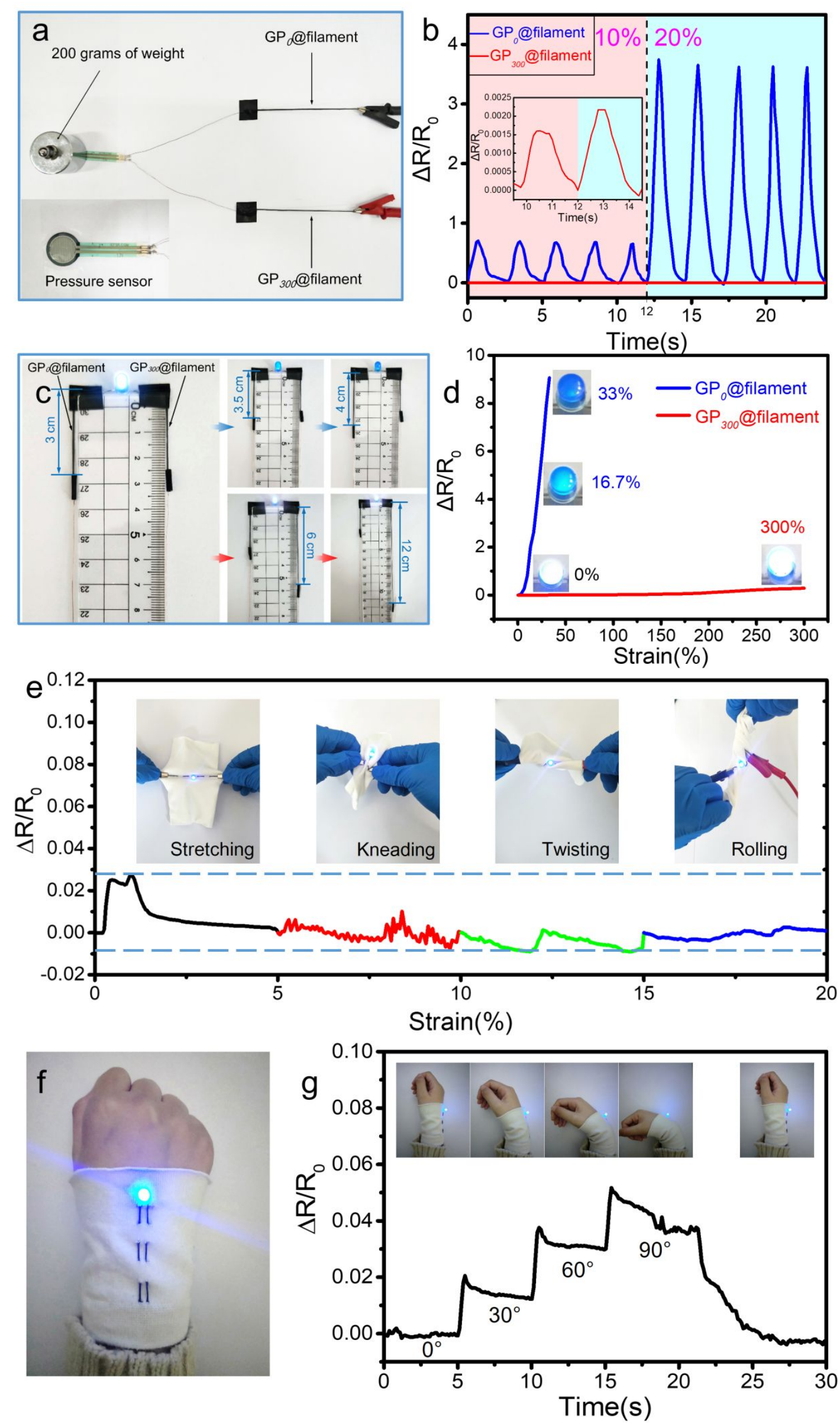
1 Figure 6. The potential applications of strain-insensitive stretchy electronics. (a) - (b)

2 Application of a simple scales using the GP $0 @$ filament and GP $300 @$ filament as the stretchable

3 electronic circuits. (c) - (d) Photographs of the LED using the GP 0 @filament and

$4 \mathrm{GP}_{300} @$ filament as electronic circuits. (e) - (g) The photographs and relative resistance

5 changes of the $\mathrm{GP}_{300} @$ filament weave into fabric to conduct signals with intricate motions (e)

6 and under robust wrist joint movements (f, g).

7 CONCLUSION

8 Bioinspired from worm micro-structure, we designed and fabricated a special 9 worm-shape GP microlayer GP ${ }_{x} @$ filament by in-situ pre-stretching wet chemistry 10 deposition. The unique GP microlayer structure endowed the GP $300 @$ filament with 11 large reversibility electrical response range of $815 \%$ strain, in which the 12 strain-insensitive range was up to $300 \%$ with a high $Q$ of 11.26 . The high $\mathrm{Q}$ provides 13 the possibility for various potential applications in deliver and transmit signals. 14 Additionally, the excellent durability over 4,000 loading/releasing cycles can serve as 15 strain-insensitive stretchy electronic in the long-term used fabric. A series of simple 16 appliances such as scales, LED systems and weaved into fabric to conduct signals 17 under robust wrist joint movements, reveal the feasibility as strain-insensitive stretchy 18 electronics. The proposed conductive filaments combine low-cost fabrication and 19 outstanding performances, providing a direct pathway for strain-insensitive stretchy 20 electronics.

21 METHODS

22 Materials. Large size scale flake graphite (lateral size $50 \mu \mathrm{m}$ ) was purchased 
1 from Qingdao Tianyuan graphite Co. LTD. Polyurethane (PU) Multifilament

2 (2500D/127f) was purchased from Zhejiang Wally Spandex Co., Ltd. N-Methyl

3 pyrrolidone (NMP) and Polyvinyl Pyrrolidone (PVP) were purchased from Aldrich.

4 Polyurethane solution (PU) with 30 wt.\% was purchased from Guangzhou Guanzhi

5 New Technology. Co., Ltd. The water used in this experiment was deionized.

7 few-layer graphene dispersion was exfoliated via the high-speed laminar flow 8 generated by a facile pressure homogenizing route according to the previous works. ${ }^{32}$,

933 Figure S1 illustrates the homogenizing mechanism of the large size scale flake graphite into few-layer graphene by a lab-scale pressure homogenizer (AH-2010, 11 ATS Engineering Inc., USA). In details, $0.5 \mathrm{~g}$ graphite was added to $100 \mathrm{~mL}$ mixture 12 solution of NMP/water (volume ratio 4:1), and $0.01 \mathrm{~g}$ polyvinyl pyrrolidone (PVP) served as the surfactant was added into the solution subsequently, following further 14 vigorous ultrasonic treatment $600 \mathrm{~W}$ for $30 \mathrm{~min}$ until achieving uniform graphite 15 dispersion. The obtained graphite dispersion was gradually injected into the homogenizer with low speed $20 \mathrm{~mL} / \mathrm{min}$, and exfoliated at a fixed pressure $50 \mathrm{Mpa}$, the collected dispersion was cooled in $20^{\circ} \mathrm{C}$ for $10 \mathrm{~min}$, and the homogenizing process was carried out eight times. After homogenization, the dispersion was centrifuged at $5000 \mathrm{rmp}$ for $10 \mathrm{~min}$ to separate the thick graphite. And the top two thirds of the dispersion were extracted and retained for use. Such method is a facile physical procedure with low cost, environment-friendly without any chemical treatment.

The evolution process from graphite to graphene in the homogenizer (axial 
1 symmetry) is shown in Figure S1b, one homogenizing cycle is composed of two

2 motions, loading and releasing pressure. Large size scale flake graphites enter a close

3 container with a high pressure and are entirely squeezed, once the valve moves to

4 leave a current-limiting gap, the pressure is instantaneously released and drove

5 graphites to dash on the impact ring. Such high gradient pressure loss and drastic

6 collision generate the coupled effect of void effect, knock-on effect, and shear effect

7 resulting in the effective exfoliation of graphite. After a series of homogenizing

8 cycles, the interlaminar force of the exfoliated graphite is gradually weakened, and

9 finally evolves into few-layer graphene nanosheets.

\section{Fabrication of conductive polyurethane filaments with worm-shape}

graphene microlayer. Graphene aqueous solution was first replaced by DMF with centrifuging the dispersion in water at $8000 \mathrm{rpm}$ for $10 \mathrm{~min}$ to collect the sediment. The obtained graphene solid was dispersed in DMF with the concentration (10 $\mathrm{mg} / \mathrm{mL}$ ). And then, the graphene solution was dropwise added into PU solution under vigorous stirring for $2 \mathrm{~h}$ until the mixed solution became homogeneous. We chose $30 \%$ as the optical case with good film-forming properties and reasonable electrical conductivity, more details can be found in Figure S2.

We in-situ deposited the laminate of a GP nanocomposite sheet conductor onto the stretched PU filaments with a worm-shape graphene microlayer on a PU filament core. The process for preparing a GP ${ }_{x} @$ filament is illustrated in Figure 1a. First, two opposite ends of a PU multifilament were attached on a frame and then the PU filament core was highly stretched under the fabrication strain $x \%$. Afterwards, the 
1 stretched PU filaments was gradually immersed into the graphene/PU inks for $5 \mathrm{~min}$

2 until entirely absorption, the soaked PU filaments were moved into the coagulation

3 water bath, the graphene/PU sheet could be in-situ deposited on the surface of the PU

4 filaments with the effect of non-solvent-induced phase separation between DMF and

5 water. After completion of solidification, the strain in the stretched PU filament was

6 gradually released to restore the non-stretched GP $@$ @filament. In our work, the PU

7 multifilament under seven pre-stretching levels ( $x$ equals to 0-300 with 50 interval)

8 were fabricated and characterized. Based on our pre-strain preliminary experiments,

$9300 \%$ case was chosen as the pre-strain limit. Once the pre-strain beyond $300 \%$

$10(350 \%$ as example in Figure S3), the microlayer of $350 \%$ case is partially burst which

11 was caused by the robust resilience force from the axial expansion of internal

12 releasing PU filaments.

13 Characterization. The morphogical structure of the graphene exfoliated by 14 pressure homogenizing, PU multifilaments, and $\mathrm{GP}_{x} @$ filament samples were 15 characterized via a transmission electronic microscopy (TEM, JEOLH-7650, Japan) 16 and scanning electron microscopy (SEM, EHT: $3.0 \mathrm{kV}$, work distance: $8.0 \mathrm{~mm}$, 17 ZEISSEVO18, Germany), respectively. Fourier transform infrared spectra (FTIR) of 18 the samples were measured on a Nicolet 5700 FTIR spectrometer (Thermo Nicolet 19 Corp, USA), the wave number range was 4000-500 cm-1. GP $300 @$ filament was rubbed 205000 cycles by LFY-109A Fiber Abrasion Tester, the particle sizes of the abrasive 21 paper in abrasion test is $\mathrm{P} 1000$ and the tension is $100 \mathrm{~N}$ which can simulate robust 22 abrasion of garment. 
All the sensing characterizations of the $\mathrm{GP}_{x} @$ filament were evaluated using the

2 System Source Meter (Models 2601B, Keithley, USA). The dynamic strain-stress

3 behaviors of the filaments were measured with a tensile testing machine (Instron

4 4206, Instron Ltd., UK) at the initial test length of $2 \mathrm{~cm}$ and at a stretch rate of 6

$5 \mathrm{~mm} / \mathrm{min}$.

$6 \quad$ The quality factor $(Q)$ of the $\mathrm{GP}_{x} @$ filament can be calculated as follows,

7

8 where $R_{0}$ represents the original electrical resistance, $\Delta R$ and $\Delta L$ indicate the change

9 of resistance and length after the corresponding strain.
10

\section{ASSOCIATED CONTENT}

\section{Supporting Information}

Figure S1: Lab-scale pressure homogenizing method preparing few-layer graphene;

Figure S2: The optical case of graphene/PU nanocomposite (GP) inks with various graphene weight ratios;

Figure S3: SEM images of GP $0 @$ filament, GP $300 @$ filament, and GP $350 @$ filament;

Figure S4: The abrasion property of $\mathrm{GP}_{300} @$ filament;

Figure S5: Models for the GP microlayer of GP $_{x} @$ filament with various pre-stretching;

Supplementary Method 1: Theoretical analysis of the filament resistance after pre-stretch;

Supplementary Method 2: Theoretical analysis of the filament resistance on strain. 
$1 \quad(\mathrm{PDF})$

2 Supplementary Movie 1: Real-time movie uses for evaluating a GP $@$ @filament and a

$3 \quad \mathrm{GP}_{300} @$ filament as a stretchy electronic circuits. (MOV)

4 Supplementary Movie 2, 3: GP $300 @$ filaments weave into fabric to conduct signals

5 under difference motions, the luminance are unaffected by fabric stretching, kneading,

6 twisting, rolling, and human joint movements. (MOV)

7

8 AUTHOR INFORMATION

9 Corresponding author

10

11

*E-mail: mwtian@qdu.edu.cn (Prof. Tian), xutailin@ustb.edu.cn (Prof. Xu), zhangxueji@ustb.edu.cn (Prof. Zhang), lijunqu@qdu.edu.cn (Prof. Qu).

\section{Author contributions}

F.S., M.T., and X.S. contributed equally to this work.

Notes

The authors declare no conflict of interest.

\section{ACKNOWLEDGMENTS}

Financial support of this work was provided by Natural Science Foundation of Shandong Province of China (ZR2018QEM004), Natural Science Foundation of China (51672141), Shandong Province College Science and Technology Plan Project (J17KA030), Technology Center of Advanced Textile Engineering Project (XJFZ/2018/01 and XJFZ/2018/05). 


\section{REFERENCES}

3 1. Schwartz, G.; Tee, B. C.; Mei, J.; Appleton, A. L.; Kim, D. H.; Wang, H.; Bao, Z.

$4 \quad$ Nat Commun 2013, 4, 1859.

5 2. Tee, B. C.; Wang, C.; Allen, R.; Bao, Z. Nat Nanotechnol 2012, 7, (12), 825-832.

6 3. Wang, C.; Hwang, D.; Yu, Z.; Takei, K.; Park, J.; Chen, T.; Ma, B.; Javey, A. Nat $7 \quad$ Mater 2013, 12, (10), 899-904.

8 4. Lipomi, D. J.; Vosgueritchian, M.; Tee, B. C.; Hellstrom, S. L.; Lee, J. A.; Fox,

9 C. H.; Bao, Z. Nat Nanotechnol 2011, 6, (12), 788-792.

10 5. Chen, Z.; Ren, W.; Gao, L.; Liu, B.; Pei, S.; Cheng, H. M. Nat Mater 2011, 10, 11 (6), 424-428.

12 6. Chen, P.; Xu, Y.; He, S.; Sun, X.; Pan, S.; Deng, J.; Chen, D.; Peng, H. Nat 13 Nanotechnol 2015, 10, (12), 1077-1083.

14 7. Chae, C.; Seo, Y. H.; Jo, Y.; Kim, K. W.; Song, W.; An, K. S.; Choi, S.; Choi, 15 Y.; Lee, S. S.; Jeong, S. ACS Appl Mater Interfaces 2015, 7, (7), 4109-4117.

8. Kim, A.; Ahn, J.; Hwang, H.; Lee, E.; Moon, J. Nanoscale 2017, 9, (18), 5773-5778.

18 9. Hu, X.; Tian, M.; Sun, B.; Qu, L.; Zhu, S.; Zhang, X. Materials Letters 2018, $19 \quad 230,148-151$.

20 10. Li, Z.; Tian, M.; Sun, X.; Zhao, H.; Zhu, S.; Zhang, X. Journal of Alloys and 21 Compounds 2019, 782, 986-994.

22 11. Lv, Z.; Tang, Y.; Zhu, Z.; Wei, J.; Li, W.; Xia, H.; Jiang, Y.; Liu, Z.; Luo, Y.; Ge, 
1 X.; Zhang, Y.; Wang, R.; Zhang, W.; Loh, X. J.; Chen, X. Adv Mater 2018, 30, (50),

21805468.

3 12. Tian, M.; Hao, Y.; Qu, L.; Zhu, S.; Zhang, X.; Chen, S. Materials Letters 2019, $4 \quad 234,101-104$.

5 13. Yamada, T.; Hayamizu, Y.; Yamamoto, Y.; Yomogida, Y.; Izadi-Najafabadi, A.;

$6 \quad$ Futaba, D. N.; Hata, K. Nat Nanotechnol 2011, 6, (5), 296-301.

7 14. Amjadi, M.; Pichitpajongkit, A.; Lee, S.; Ryu, S.; Park, I. Acs Nano 2014, 8, (5), $8 \quad 5154-5163$.

9 15. Lu, Y.; Tian, M.; Sun, X.; Pan, N.; Chen, F.; Zhu, S.; Zhang, X.; Chen, S.; 10 Manufacturing. Composites Part A: Applied Science 2019, 117, 202-210.

11 16. Matsuhisa, N.; Inoue, D.; Zalar, P.; Jin, H.; Matsuba, Y.; Itoh, A.; Yokota, T.; 12 Hashizume, D.; Someya, T. Nat Mater 2017, 16, (8), 834-840.

13 17. Zhao, H.; Tian, M.; Hao, Y.; Qu, L.; Zhu, S.; Chen, S. Journal of materials 14 science 2018, 53, (13), 9504-9520.

15 18. Zhang, X.; Shi, M. Journal of hazardous materials 2019, 365, 9-15.

16 19. Park, M.; Im, J.; Shin, M.; Min, Y.; Park, J.; Cho, H.; Park, S.; Shim, M. B.; Jeon, 17 S.; Chung, D. Y.; Bae, J.; Park, J.; Jeong, U.; Kim, K. Nat Nanotechnol 2012, 7, (12), $18 \quad 803-809$.

19 20. Moon, S.; Park, H. K.; Song, J. H.; Cho, S.; Kim, J. C.; Kim, J.; Hwang, H.; Kim, 20 H. S.; Jeong, U. Adv Mater 2018, 30, (32), 1801408.

21 21. Ho, M. D.; Liu, Y.; Dong, D.; Zhao, Y.; Cheng, W. Nano Lett 2018, 18, (6), $22 \quad 3593-3599$. 
1 22. Li, C.-F.; Li, W.; Zhang, H.; Jiu, J.; Yang, Y.; Li, L.; Gao, Y.; Liu, Z.-Q.;

2 Suganuma, K. J. ACS Appl Mater Interfaces 2018, 11, (3), 3231-3240.

3 23. Liu, Z.; Fang, S.; Moura, F. A.; Ding, J.; Jiang, N.; Di, J.; Zhang, M.; Lepró, X.;

4 Galvão, D.; Haines, C. J. S. Science 2015, 349, (6246), 400-404.

5 24. Tian, M.; Chi, S.; Qu, L.; Chen, S.; Ran, J.; Zhu, S.; Zhang, X. Iranian Polymer 6 Journal 2019, 27, (2), 115-124.

7 25. Cervantes-Uc, J. M.; Espinosa, J. I. M.; Cauich-Rodríguez, J. V.; Ávila-Ortega,

8 A.; Vázquez-Torres, H.; Marcos-Fernández, A.; San Román, J. Polymer Degradation 9 and Stability 2009, 94, (10), 1666-1677.

10 26. Shi, G.; Zhao, Z.; Pai, J.-H.; Lee, I.; Zhang, L.; Stevenson, C.; Ishara, K.; Zhang, 11 R.; Zhu, H.; Ma, J. Advanced Functional Materials 2016, 26, (42), 7614-7625.

27. Xu, F.; Zhu, Y. Adv Mater 2012, 24, (37), 5117-5122.

28. Ge, J.; Yao, H. B.; Wang, X.; Ye, Y. D.; Wang, J. L.; Wu, Z. Y.; Liu, J. W.; Fan, F. J.; Gao, H. L.; Zhang, C. L.; Yu, S. H. Angew Chem Int Ed Engl 2013, 52, (6), $1654-1659$.

29. Lee, G.; Lee, S. G.; Chung, Y.; Bae, G. Y.; Lee, S.; Ryu, S.; Cho, K. Advanced Electronic Materials 2016, 2, (8), 1600158.

30. Shin, M. K.; Oh, J.; Lima, M.; Kozlov, M. E.; Kim, S. J.; Baughman, R. H. Adv Mater 2010, 22, (24), 2663-2667.

31. Lee, P.; Ham, J.; Lee, J.; Hong, S.; Han, S.; Suh, Y. D.; Lee, S. E.; Yeo, J.; Lee, S. S.; Lee, D.; Ko, S. H. Advanced Functional Materials 2014, 24, (36), 5671-5678.

32. Arao, Y.; Mizuno, Y.; Araki, K.; Kubouchi, M. Carbon 2016, 102, 330-338. 
1 33. Arao, Y.; Mori, F.; Kubouchi, M. Carbon 2017, 118, 18-24. 